\title{
The conformational distribution in diphenylmethane determined by nuclear magnetic resonance spectroscopy of a sample dissolved in a nematic liquid crystalline solvent
}

\author{
G. Celebre and G. De Luca \\ Dipartimento di Chimica, Universita della Calabria, 87030 Arcavacata di Rende (Cs), Italy \\ J. W. Emsley and E. K. Foord \\ Chemistry Department, University of Southampton, Southampton SO17 1BJ, United Kingdom \\ M. Longeri, F. Lucchesini, ${ }^{\text {a) }}$ and G. Pileio \\ Dipartimento di Chimica, Universita della Calabria, 87030 Arcavacata di Rende (Cs), Italy
}

(Received 10 October 2002; accepted 3 January 2003)

\begin{abstract}
The deuterium decoupled, proton nuclear magnetic resonance spectrum of a sample of diphenylmethane- $d_{3}$ dissolved in a nematic liquid crystalline solvent has been analyzed to yield a set of dipolar couplings, $D_{i j}$. These have been used to test models for the conformational distribution generated by rotation about the two ring- $\mathrm{CH}_{2}$ bonds through angles $\tau_{1}$ and $\tau_{2}$. Conformational distributions, particularly when obtained from a quantum chemistry calculation, are usually described in terms of the potential energy surface, $V\left(\tau_{1}, \tau_{2}\right)$, which is then used to define a probability density distribution, $P\left(\tau_{1}, \tau_{2}\right)$. It is shown here that when attempting to obtain $P\left(\tau_{1}, \tau_{2}\right)$ from experimental data it can be an advantage to do this directly without going through the intermediate step of trying to characterize $V\left(\tau_{1}, \tau_{2}\right)$. When applied to diphenylmethane this method shows that the dipolar couplings are consistent with a conformational distribution centered on $\tau_{1}=\tau_{2}=56.5 \pm 0.5^{\circ}$, which is close to the values calculated for an isolated molecule of $57.0^{\circ}$, and significantly different from the asymmetric structure found in the crystalline state. (C) 2003 American Institute of Physics. [DOI: 10.1063/1.1555631]
\end{abstract}

\section{INTRODUCTION}

Molecules with internal bond rotational possibilities usually adopt a single conformation in the solid state, but have a conformational distribution in liquids and gases. The possibility exists that the global energy minimum changes in structure between the three phases because of the influence of intermolecular forces. To investigate this possibility it is necessary to determine the structure and conformation in each phase. The structure in the solid state can be obtained with high precision, and relatively easily by $\mathrm{x}$-ray or neutron diffraction, if the compound forms good crystals at an accessible temperature. These methods may also be applied to molecules of large or small molar mass. For the gaseous state the experimental methods are more limited in their range of application, and there may also be severe experimental difficulties. An alternative is to calculate an energy surface by solving Schrödinger's equation for the electronic wave functions of an isolated molecule. This approach always involves some degree of approximation, which varies with the number of atoms and electrons in the molecule, and at the present time there is considerable interest in investigating the validity of the commonly available computational methods, together with their associated computer programs.

For the liquid state, which chemically and biologically is generally the most interesting, the available experimental

\footnotetext{
${ }^{a}$ Present address: Dipartimento di Chimica e Tecnologie Farmaceutiche e Alimentari, Università di Genova, 16147 Genova, Italy.
}

methods are either of limited applicability, or are at an early stage of development. Here we present a study of diphenylmethane by the method which relies on being able to obtain partially-averaged dipolar couplings, $D_{i j}$, between pairs of nuclei by analysing the nuclear magnetic resonance (NMR) spectrum of a sample dissolved in a liquid crystalline phase. This so-called LXNMR method has a wide range of applicability, and has been used for small, rigid molecules such as benzene ${ }^{1}$ in order to make a comparison at high precision between structures obtained in solid, liquid, and gaseous phases, and for molecules as large as ubiquitin to obtain coarse grained structures of this and similar proteins in their folded states. ${ }^{2,3}$

A study of the conformation of diphenylmethane is important because it is the simplest case of linkage of two aromatic rings through a methylene group. Such a linkage exists, for example, in trimethoprim, an important therapeutic agent, and in some recently synthesized liquid crystals. ${ }^{4}$

Diphenylmethane also presents several interesting challenges to the LXNMR method, which we will address here. First, the proton spectrum of a sample dissolved in a liquid crystalline solvent is that of 12 interacting spins, and it is too complex to be analyzed without following some spectral analysis simplification strategy. Also, the question has to be asked of whether it is necessary to analyze the spectrum of the fully protonated molecule or would a smaller data set, obtained from the analysis of selectively deuteriated isotopomers, be sufficiently large to test models for the conformational distribution? The approach adopted here is to pre- 


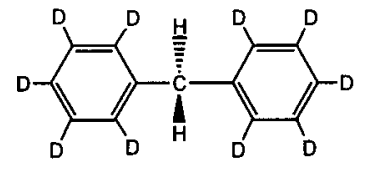

1

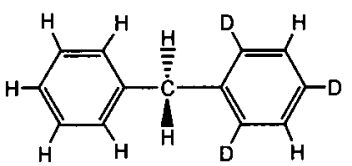

3

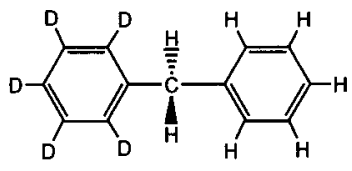

2

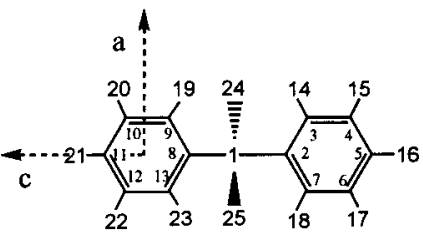

FIG. 1. Structure of isotopomers and atomic labeling of diphenylmethane.

pare suitably deuteriated samples and to analyze the simpler, ${ }^{1} \mathrm{H}-\left\{{ }^{2} \mathrm{H}\right\}$ spectra.

Second, in order to derive a conformational distribution from a set of $D_{i j}$ it is necessary to have a theoretical model to treat the coupling between internal and reorientational motions, and a parameterized functional form for the conformational surface which is then brought into agreement with the experiment data by varying the parameters in a systematic way.

It is usual to describe the conformational surface by a potential energy function, most often a Fourier series, plus a term to take into account steric, repulsive interactions. Such an approach is possible for diphenylmethane, but we will show that it is simpler to use a direct probability description of the conformational distribution. This new probability approach is of a general nature, and has particular advantages for cases where rotations about two bonds (such as diphenylmethane) or more are of a cooperative nature.

Third, diphenylmethane has been studied by $\mathrm{x}$-ray diffraction, ${ }^{5}$ and by several quantum chemistry methods, 6,7 and these results act as a useful set of criteria to judge the success of the LXNMR method.

\section{EXPERIMENT}

A general way of extracting dipolar couplings between protons is to simplify the spectrum by replacement of ${ }^{1} \mathrm{H}$ by ${ }^{2} \mathrm{H}$, followed by deuterium decoupling. To apply this method to diphenylmethane required the synthesis of compounds 1-3 (Fig. 1).

\section{A. Synthesis of isotopically labeled samples}

\section{Synthesis of compound 1}

Diphenylmethane $(0.50 \mathrm{~g}, 2.97 \mathrm{mmol})$ and benzene- $d_{6}$ $(10 \mathrm{ml})$ were introduced into a three-necked flask fitted with a calcium chloride guard tube and a water-cooled condenser. The flask was flushed with nitrogen; a $1 \mathrm{M}$ solution of ethylaluminum dichloride in hexanes $(2 \mathrm{ml})$ (Aldrich) was added and the solution refluxed for $1 \mathrm{~h}$ under stirring. The solution was allowed to cool, opened to the air, and $\mathrm{D}_{2} \mathrm{O}$ added until the yellow color in the organic layer disappeared. The organic layer was separated, dried over magnesium sulphate, and the deuterated benzene removed by rotary evaporation. The crude product, which was about $80 \%$ deuterated, was used without further purification.

\section{Synthesis of compound 2}

Benzyl chloride $(3.80 \mathrm{~g}, 30.0 \mathrm{mmol})$ and benzene- $d_{6}(15$ $\mathrm{ml})$ in a three-necked flask equipped with a calcium chloride guard tube, a magnetic stirrer and a water-cooled condenser were cooled with an ice bath under stirring and tin (IV) chloride $(0.40 \mathrm{~g}, 1.53 \mathrm{mmol})$ was added slowly in five equal amounts. After stirring for $15 \mathrm{~min} \mathrm{D}_{2} \mathrm{O}$ was added (5 $\times 10 \mathrm{ml}$ ) to decompose the catalyst. The mixture was washed with water $(2 \times 10 \mathrm{ml})$ and $1 \mathrm{M} \mathrm{HCl}(2 \times 10 \mathrm{ml})$, the organic layer was separated and dried over magnesium sulphate. Volatile impurities were removed at $100{ }^{\circ} \mathrm{C} / 3$ Torr, leaving behind the desired diphenylmethane- $d_{5} 2$.

\section{Synthesis of compound 3}

a. Preparation of bromobenzene-2,4,6- $d_{3}$. Aniline hydrochloride $(6.00 \mathrm{~g}, 47.4 \mathrm{mmol})$ and $\mathrm{D}_{2} \mathrm{O}$ (12.00 g) (SigmaAldrich $D=99.9 \%$ ) were introduced into a flask equipped with a stirrer and a water-cooled condenser. The solution was heated at reflux under nitrogen for $24 \mathrm{~h}$ and the water was removed under reduced pressure. The procedure was repeated four times, then the aniline hydrochloride-2,4,6- $d_{3}$ was dissolved in $10 \mathrm{ml} 10 \% \mathrm{NaOH}$ solution, the base extracted into $\mathrm{Et}_{2} \mathrm{O}(3 \times 50 \mathrm{ml})$ and dried over sodium sulphate. Removal of the solvent left the aniline-2,4,6- $d_{3}(3.23$ g, $52 \%$ yield, ${ }^{2} \mathrm{H}=98 \%$ ).

The aniline-2,4,6- $d_{3}(3.0 \mathrm{~g}, 31.2 \mathrm{mmol})$ was dissolved in aqueous $8.83 \mathrm{M} \mathrm{HBr}(22 \mathrm{ml})$ and diazotized with $12.2 \mathrm{ml}$ of aqueous $2.5 \mathrm{M}$ sodium nitrite at $T<5{ }^{\circ} \mathrm{C}$. This diazonium salt solution was poured into a three-necked flask equipped with a stirrer and a water-cooled condenser containing copper (I) bromide $(6.34 \mathrm{~g}, 44.2 \mathrm{mmol})$ and $18 \mathrm{ml}$ conc. $\mathrm{HBr}$. The mixture was heated at reflux for $2 \mathrm{~h}$, steam distilled, and the product extracted into $\mathrm{Et}_{2} \mathrm{O}$. The organic layer was washed with $10 \%$ sodium hydroxide solution, then with water up to neutrality, dried over sodium sulphate, and the solvent removed by rotary evaporation. The crude product was distilled $\left(53{ }^{\circ} \mathrm{C} / 23\right.$ Torr $)$ to yield bromobenzene-2,4,6- $d_{3}$ (2.10 g, 50\% yield).

b. Preparation of diphenylmethanol-2,4,6- $d_{3}$. Bromobenzene-2,4,6- $d_{3}(2.00 \mathrm{~g}, 12.6 \mathrm{mmol})$ in anhydrous $\mathrm{Et}_{2} \mathrm{O}(35$ $\mathrm{ml})$ was cooled to $0{ }^{\circ} \mathrm{C}$ under nitrogen. $n$-Butyl lithium in hexanes (1.68 M, $8.3 \mathrm{ml})$ was added slowly, dropwise, keeping the temperature below $2{ }^{\circ} \mathrm{C}$. The mixture was stirred at room temperature for $1 \mathrm{~h}$ after which was cooled again at $0{ }^{\circ} \mathrm{C}$ and benzaldehyde $(1.48 \mathrm{~g}, 14.0 \mathrm{mmol})$ added. The cooling bath was removed and the mixture left at room temperature for $2.5 \mathrm{~h}$. A solution of $\mathrm{NH}_{4} \mathrm{Cl}(10 \mathrm{ml})$ was added, the organic layer separated, washed with water and dried over sodium sulphate. Removal of the solvent left the crude product $(2.3 \mathrm{~g}, 98 \%$ yield), which was used in the next stage without purification.

c. Preparation of benzophenone-2,4,6- $d_{3}$. Diphenylmethanol-2,4,6- $d_{3}(2.35 \mathrm{~g}, 12.5 \mathrm{mmol})$ in dry dichloromethane $(17 \mathrm{ml})$ was added to a stirred mixture of pyri- 


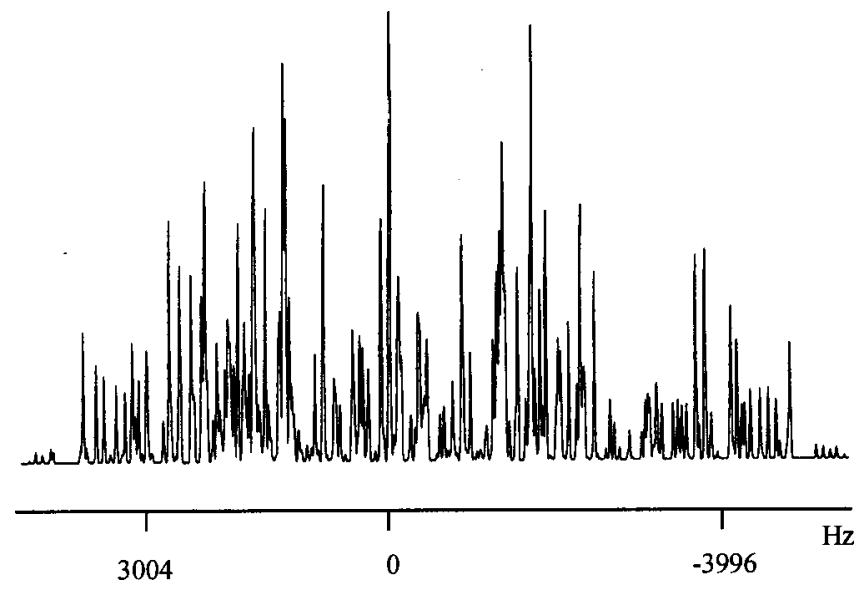

FIG. 2. $200 \mathrm{MHz}{ }^{1} \mathrm{H}-\left\{{ }^{2} \mathrm{H}\right\}$ NMR spectrum of a sample of diphenylmethane- $d_{5}$ (compound 2) dissolved in the nematic solvent ZLI 1132 at 300 $\mathrm{K}$.

dinium chlorochromate $(4.13 \mathrm{~g}, 19.1 \mathrm{mmol})$ and sodium acetate $(0.31 \mathrm{~g}, 3.8 \mathrm{mmol})$ in dry dichloromethane $(17 \mathrm{ml})$. The mixture was stirred for $2 \mathrm{~h}$ at room temperature and then dry $\mathrm{Et}_{2} \mathrm{O}(35 \mathrm{ml})$ was added. The organic phase was separated and the solvent was removed using a rotary evaporator to leave an oily product, which was distilled under reduced pressure $\left(105^{\circ} \mathrm{C} / 0.9\right.$ Torr $)$ to yield the product $(1.35 \mathrm{~g}, 58 \%$ yield).

d. Preparation of diphenylmethane-2,4,6- $d_{3}$. A solution of benzophenone-2,4,6- $d_{3}(1.34 \mathrm{~g}, 7.23 \mathrm{mmol})$, hydrazine hydrate $(1.24 \mathrm{ml}, 0.0247 \mathrm{~mol})$, and $\mathrm{KOH}(1.85 \mathrm{~g}, 0.0330$ mol) in triethylene glycol $(4 \mathrm{ml})$ was refluxed for $2 \mathrm{~h}$ with vigorous stirring. Pentane $(20 \mathrm{ml})$ was added at room temperature and the organic layer separated, washed with water and dried with sodium sulphate. The solvent was removed and the residue distilled under reduced pressure $\left(69^{\circ} \mathrm{C} / 2\right.$ Torr $)$ to give the desired product $3(0.586 \mathrm{~g}, 47 \%$ yield).

All the compounds have been characterized by NMR and the isotopic purity of compounds $\mathbf{2}$ and $\mathbf{3}$ was determined to be $>95 \%$.

\section{B. NMR experiments}

Samples of compounds 1-3 were prepared by dissolving approximately $10 \%$ by weight in the nematic mixture ZLI 1132, obtained from Merck (UK) Ltd. The ${ }^{1} \mathrm{H}-\left\{{ }^{2} \mathrm{H}\right\}$, and ${ }^{2} \mathrm{H}$ spectra were obtained with a Bruker MSL 200 spectrometer at $300 \mathrm{~K}$ on samples contained in $5 \mathrm{~mm}$ o.d. sample tubes using a probe with a $7 \mathrm{~mm}$ horizontal solenoid coil. Deuterium decoupling was achieved using a single pulse at the center of the deuterium spectrum and applied only during the acquisition period. A delay of $5 \mathrm{~s}$ between pulses was allowed in order to minimize the heating effects of the deuterium irradiation.

\section{ANALYSIS OF SPECTRA}

The ${ }^{1} \mathrm{H}-\left\{{ }^{2} \mathrm{H}\right\}$ spectra of $\mathbf{2}$ and $\mathbf{3}$ are complex, as shown in Figs. 2 and 3, and to analyze them by the conventional approach requires that a trial set of $D_{i j}$ are obtained which can be used to simulate spectra which are close to those

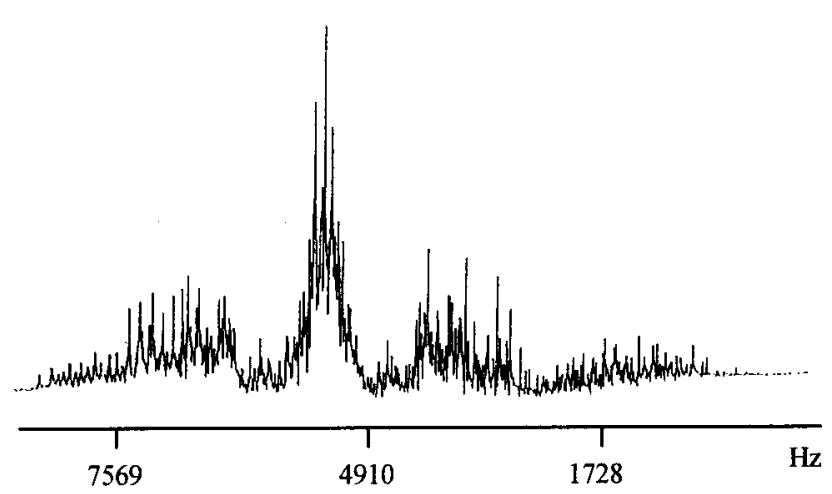

FIG. 3. $200 \mathrm{MHz}{ }^{1} \mathrm{H}-\left\{{ }^{2} \mathrm{H}\right\}$ NMR spectrum of a sample of diphenylmethane- $d_{3}$ (compound $\mathbf{3}$ ) dissolved in the nematic solvent ZLI 1132 at 300 K.

observed. The first step in obtaining the "good" set of $D_{i j}$ was to record a ${ }^{1} \mathrm{H}-\left\{{ }^{2} \mathrm{H}\right\}$ spectrum of $\mathbf{1}$, which is a doublet with a separation of $3\left|D_{24,25}\right|$. To proceed further, the ${ }^{2} \mathrm{H}$ spectrum of $\mathbf{2}$ was recorded. This consists of just four lines: an outer pair from the deuterium at position 21, with combined intensity of unity, and a separation $\Delta \nu_{p}$, and an inner pair, intensity 4 , separation $\Delta \nu_{o, m}$ from the deuteriums at positions 19, 20, 22, and 23. The deuteriums at positions 19 and 23 are expected to be equivalent and to give one quadrupolar doublet, and the equivalent pair of deuteriums at positions 20 and 22 should in principle give another, separate doublet. The observation that only one doublet is observed shows that the chemical shift difference between these four deuteriums is negligible, and that the vectors $\mathbf{r}_{\mathbf{9}, \mathbf{1 9}}$ and $\mathbf{r}_{\mathbf{1 2 , 2 2}}$ are collinear, and so too are $\mathbf{r}_{10,20}$ and $\mathbf{r}_{13,23}$.

The quadrupolar splittings are related to local order parameters, ${ }^{8,9} S_{\alpha \beta}^{R}$, by

$$
\begin{aligned}
\Delta \nu_{p}= & (3 / 2) q_{\mathrm{CD}} S_{c c}^{R}, \\
\Delta \nu_{o, m}= & (3 / 4) q_{\mathrm{CD}}\left[S_{c c}^{R}\left(3 \cos ^{2} \theta_{o, m}-1\right)\right. \\
& \left.+\left(S_{a a}^{R}-S_{b b}^{R}\right) \sin ^{2} \theta_{o, m}\right],
\end{aligned}
$$

where it has been assumed that the quadrupolar tensors for the deuteriums at the three ring positions are equal and are each axially-symmetric about the bond directions. The axes $a$ and $c$ are fixed in the ring plane, as shown in Fig. 1, and $b$ is the normal to the plane. The angle between the C-D directions and the $c$ axis, $\theta_{o, m}$, is taken to be $60^{\circ}$. The quadrupolar coupling constant, $q_{\mathrm{CD}}$, is assumed to have the value of $185 \mathrm{kHz}$. The signs of the quadrupolar splittings are not determined by the experiments, and so there are four possible solutions for the two order parameters. These are used to predict the dipolar couplings between protons in the ring of 2 from

$$
\begin{aligned}
D_{i j}= & -\left(\mu_{0} \gamma_{H}^{2} h / 32 \pi^{3} r_{i j}^{3}\right)\left[S_{c c}^{R}\left(3 \cos ^{2} \theta_{i j c}-1\right)\right. \\
& \left.+\left(S_{a a}^{R}-S_{b b}^{R}\right)\left(\cos ^{2} \theta_{i j a}-\cos ^{2} \theta_{i j b}\right)\right],
\end{aligned}
$$

where $\mu_{0}$ is the permeability of free space and $\gamma_{H}$ is the gyromagnetic ratio for the proton.

The ${ }^{2} \mathrm{H}$ spectrum given by 3 is shown in Fig. 4, and has fine structure on the peaks from dipolar coupling to the protons as shown in the expanded section. The triplet structure 


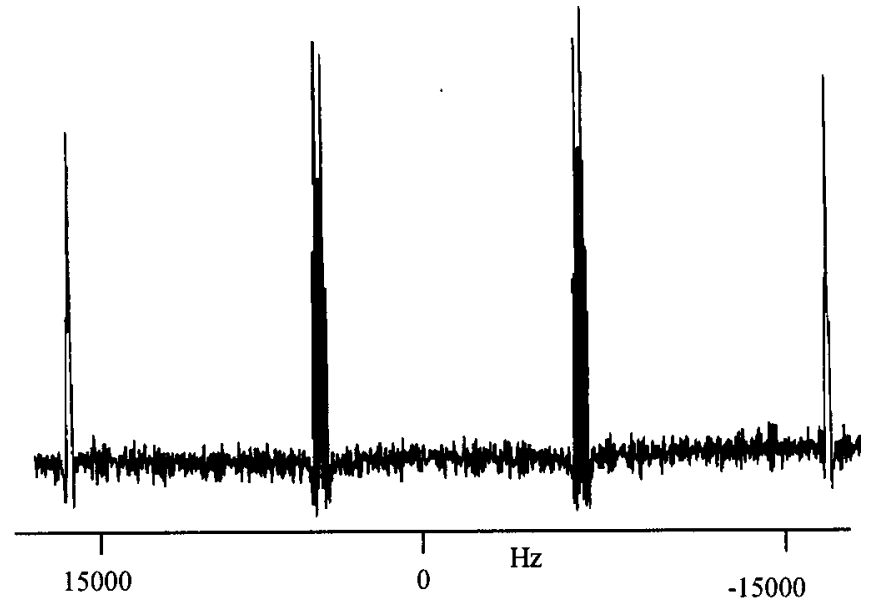

(a)

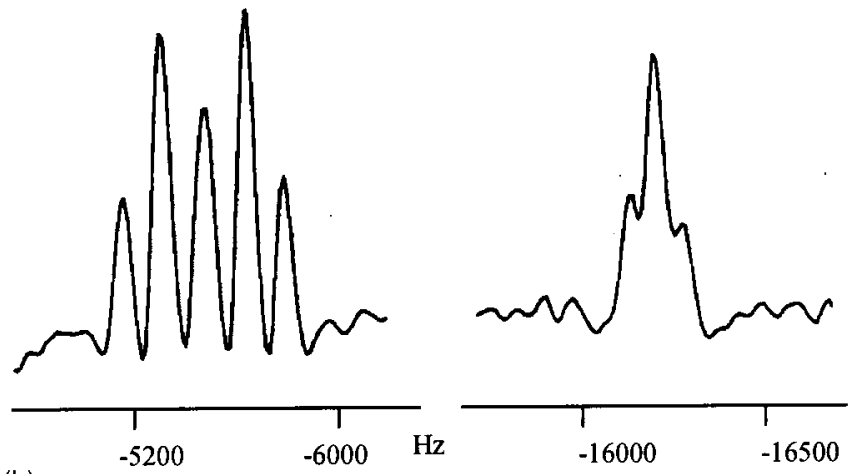

(b)

FIG. 4. $30.7 \mathrm{MHz}$ spectrum from the deuteriums in a sample of diphenylmethane- $d_{3}$ (compound 3) dissolved in the nematic solvent ZLI 1132 at $300 \mathrm{~K}$ : (top) normal spectrum and, (bottom) expanded sections where the fine structure of multiplets is shown.

of the peak from deuterium at position 16 is from coupling to the protons at positions 15 and 17 , and this allows a value to be obtained for $\left|D_{15,16}\right|$. The peaks from deuterium at position 14 (and 18) show a doublet from coupling to proton at position 15, and a triplet from coupling to protons at positions 24 and 25, and these allow values to be obtained of $\left|D_{14,15}\right|$ and $\left|D_{14,24}\right|$.

These experiments enabled good estimates to be made of all the dipolar couplings within the $\mathrm{C}_{6} \mathrm{H}_{5} \mathrm{CH}_{2}$-group, with the exception of $D_{15,24}$, and this was estimated by comparison with the set of data obtained for benzyl chloride dissolved in the same liquid crystalline solvent. ${ }^{10}$ With this set of starting values it was possible to analyze the ${ }^{1} \mathrm{H}-\left\{{ }^{2} \mathrm{H}\right\}$ spectrum of 2 shown in Fig. 2 by the method of Celebre et $a l .{ }^{11}$ to give the data in Table I. These $D_{i j}$ were then used as starting parameters in the analysis of the ${ }^{1} \mathrm{H}-\left\{{ }^{2} \mathrm{H}\right\}$ spectrum of compound $\mathbf{3}$, which is shown in Fig. 3, to give the data in Table II.

\section{CONFORMATIONAL ANALYSIS: THEORY}

\section{A. General}

The $D_{i j}$ are averages over all the normal modes of the molecule relative to the applied magnetic field, which in the present case is parallel to the mesophase director, $\mathbf{n}$. Thus, ${ }^{12}$
TABLE I. Chemical shifts $\Delta \nu_{i j}$, and dipolar couplings, $D_{i j}$, obtained from the analysis of the $200 \mathrm{MHz}{ }^{1} \mathrm{H}-\left\{{ }^{2} \mathrm{H}\right\}$ spectrum of a sample of diphenylmethane- $d_{5}$ (compound 2) dissolved in ZLI 1132.

\begin{tabular}{ccc}
\hline \hline$i j$ & $J_{i j} / \mathrm{Hz}^{\mathrm{a}}$ & $D_{i j} / \mathrm{Hz}^{\mathrm{b}}$ \\
\hline 19,20 & 8.00 & -994 \\
19,21 & 2.00 & -146 \\
19,22 & $\cdots$ & -38 \\
19,23 & 2.00 & -14 \\
19,24 & $\cdots$ & -429 \\
20,21 & 8.00 & -311 \\
20,22 & 2.00 & -15 \\
20,24 & $\cdots$ & -83 \\
21,24 & $\cdots$ & -54 \\
24,25 & $\cdots$ & 2068 \\
& & $\Delta \nu_{i j} / \mathrm{Hz}$ \\
19,21 & & -27 \\
20,21 & & -44 \\
24,21 & & -614 \\
\hline
\end{tabular}

${ }^{a}$ W. Brugel, NMR Spectra and Chemical Structure (Academic, New York, 1967).

${ }^{b}$ The spectrum was simulated with a total rms error of $7 \mathrm{~Hz}$. The error on each NMR parameter is estimated to be $\pm 0.5 \mathrm{~Hz}$

$$
D_{i j} \equiv \widetilde{D}_{i j \mathrm{ZZ}}=\int P_{\mathrm{LC}}\left(\tau_{k}, v_{m}\right) D_{i j}\left(\tau_{k}, v_{m}\right) d \tau_{k} d v_{m},
$$

where $P_{\mathrm{LC}}\left(\tau_{k}, v_{m}\right)$ is the probability in the liquid crystal phase that the molecule is in a conformation defined by the bond rotation angles $\tau_{k}$, and a vibrational state defined by $v_{m}$. A vibrational state is defined here as involving only small amplitude displacements of nuclei, and does not therefore include bond rotational motion. The definition of $D_{i j}\left(\tau_{k}, v_{m}\right)$ is

TABLE II. Chemical shifts, $\Delta \nu_{i j}$, and dipolar couplings, $D_{i j}$, obtained from the analysis of the $200 \mathrm{MHz}{ }^{1} \mathrm{H}-\left\{{ }^{2} \mathrm{H}\right\}$ spectrum of a sample of diphenylmethane- $d_{3}$ (compound 3) dissolved in ZLI 1132; the calculated values were obtained by the probabilistic approach without (column A) and with (column B) vibrational corrections to the dipolar couplings.

\begin{tabular}{cccr}
\hline \hline$i, j$ & \multicolumn{3}{c}{$D_{i j} / \mathrm{Hz}$} \\
& \multicolumn{3}{c}{${ }^{2}$ Calculated } \\
& Experiment & \multicolumn{1}{c}{$B$} \\
\hline 15,17 & $-15.0 \pm 0.1$ & -70.0 & -14.1 \\
15,19 & $-70.0 \pm 0.1$ & -40.6 & -70.2 \\
15,20 & $-40.7 \pm 0.1$ & -36.4 & -36.3 \\
15,21 & $-35.7 \pm 0.1$ & -73.0 & -73.6 \\
15,24 & $-73.0 \pm 0.1$ & -897.0 & -896.5 \\
19,20 & $-897.1 \pm 0.1$ & -133.2 & -135.0 \\
19,21 & $-133.2 \pm 0.1$ & -35.2 & -35.8 \\
19,22 & $-35.4 \pm 0.1$ & -15.1 & -14.1 \\
19,23 & $-15.0 \pm 0.1$ & -391.9 & -391.9 \\
19,24 & $-391.9 \pm 0.1$ & -281.1 & -281.2 \\
20,21 & $-281.1 \pm 0.1$ & -50.1 & -51.1 \\
21,24 & $-48.6 \pm 0.1$ & 1847.5 & 1847.5 \\
24,25 & $1847.5 \pm 0.1$ & 0.4 & 1.0 \\
$R / \mathrm{Hz}$ & & & \\
\multicolumn{5}{c}{} & $\Delta \nu_{i j} / \mathrm{Hz}$ & & \\
15,21 & $-18.0 \pm 0.1$ & & \\
19,21 & $-50.0 \pm 0.1$ & & \\
24,21 & $-628.8 \pm 0.1$ & & \\
\hline \hline
\end{tabular}




$$
\begin{aligned}
D_{i j}\left(\tau_{k}, v_{m}\right)= & \left(\frac{\mu_{0}}{4 \pi}\right) \frac{\gamma_{i} \gamma_{j} h}{8 \pi^{2}\left(r_{i j}^{\left.\tau_{k}, v_{m}\right)^{3}}\right.}\left[S_{z z}\left(\tau_{k}, v_{m}\right)\right. \\
& \times\left(3 \cos ^{2} \theta_{i j z}^{k, m}-1\right)+\left(S_{x x}\left(\tau_{k}, v_{m}\right)\right. \\
& \left.\left.-S_{y y}\left(\tau_{k}, v_{m}\right)\right)\left(\cos ^{2} \theta_{i j x}^{k, m}-\cos ^{2} \theta_{i j y}^{k, m}\right)\right],
\end{aligned}
$$

where $S_{z z}\left(\tau_{k}, v_{m}\right)$, etc. vary with the conformation and vibrational state. These order parameters are for principal axes, and these axes will also change their orientation in different conformational and vibrational states.

It is usual to assume that the dependence of the order parameters on the vibrational state ${ }^{13}$ is much smaller than their dependence on the bond rotational angles, and can be neglected to a good approximation. Similarly it is argued that the dependence of $D_{i j}\left(\tau_{k}, v_{m}\right)$ on $v_{m}$ can also be neglected when the main interest is in trying to characterize $P_{\mathrm{LC}}\left(\tau_{k}\right)$, the conformational distribution, from a set of experimental dipolar couplings. To support this view it can be noted that vibrational corrections to $D_{i j}$ in rigid molecules are usually $<5 \%$, whereas the dependence of $D_{i j}\left(\tau_{k}, v_{m}\right)$ on $\tau_{k}$ is often $>100 \%$. Of course, the main reason for neglecting the effects of vibrations when large amplitude motion is also present is the difficulty of their calculation. Here the effects of vibrations will be neglected at first, but later an approximate method for their inclusion will be explored.

Neglecting vibrational motion reduces Eqs. (4) and (5) to

$$
D_{i j}=\int P_{\mathrm{LC}}\left(\tau_{k}\right) D_{i j}\left(\tau_{k}\right) d \tau_{k}
$$

and

$$
\begin{aligned}
D_{i j}\left(\tau_{k}\right)= & -\left(\frac{\mu_{0}}{4 \pi}\right) \frac{\gamma_{i} \gamma_{j} h}{8 \pi^{2}\left(r_{i j}^{\tau_{k}}\right)^{3}}\left[S_{z z}\left(\tau_{k}\right)\left(3 \cos ^{2} \theta_{i j z}^{k}-1\right)\right. \\
& \left.+\left(S_{x x}\left(\tau_{k}\right)-S_{y y}\left(\tau_{k}\right)\right)\left(\cos ^{2} \theta_{i j x}^{k}-\cos ^{2} \theta_{i j y}^{k}\right)\right] .
\end{aligned}
$$

It is tempting to assume that the dependence of $S_{\alpha \alpha}\left(\tau_{k}\right)$ on $\tau_{k}$ can also be neglected, but although this is usually done by those using dipolar couplings to study protein structures it is likely to lead to major errors in the function $P_{\mathrm{LC}}\left(\tau_{k}\right)$ and the structures derived when there is an appreciable degree of intramolecular motion present in the molecule.

To proceed further, a probability, $P_{\mathrm{LC}}\left(\beta, \gamma, \tau_{k}\right)$, that the molecule is at an orientation with the director defined by the polar angles $\beta$ and $\gamma$ whilst in a conformation described by the set $\tau_{k}$ is introduced. It is defined in terms of a mean potential, $U_{\mathrm{LC}}\left(\beta, \gamma, \tau_{k}\right)$, such that

$$
P_{\mathrm{LC}}\left(\beta, \gamma, \tau_{k}\right)=Z^{-1} \exp \left[-U_{\mathrm{LC}}\left(\beta, \gamma, \tau_{k}\right) / k_{B} T\right]
$$

with

$$
Z=\int \exp \left[-U_{\mathrm{LC}}\left(\beta, \gamma, \tau_{k}\right) / k_{B} T\right] \sin \beta d \beta d \gamma d \tau_{k} .
$$

The mean potential can be divided into a part, $U_{\text {ext }}\left(\beta, \gamma, \tau_{k}\right)$, which vanishes for an isotropic phase, and a part, $U_{\text {int }}\left(\tau_{k}\right)$, which does not,

$$
U_{\mathrm{LC}}\left(\beta, \gamma, \tau_{k}\right)=U_{\text {ext }}\left(\beta, \gamma, \tau_{k}\right)+U_{\text {int }}\left(\tau_{k}\right) .
$$

The function $P_{\mathrm{LC}}\left(\tau_{k}\right)$ is related to $P_{\mathrm{LC}}\left(\beta, \gamma, \tau_{k}\right)$,

$$
\begin{aligned}
P_{\mathrm{LC}}\left(\tau_{k}\right)= & \int \mathrm{P}_{\mathrm{LC}}\left(\beta, \gamma, \tau_{k}\right) \sin \beta d \beta d \gamma \\
= & Z^{-1} \exp \left[-U_{\mathrm{int}}\left(\tau_{k}\right) / k_{B} T\right] \\
& \times \int \exp \left[-U_{\text {ext }}\left(\beta, \gamma, \tau_{k}\right) / k_{B} T\right] \sin \beta d \beta d \gamma .
\end{aligned}
$$

The order parameters $S_{\alpha \alpha}\left(\tau_{k}\right)$ are obtained as

$$
\begin{aligned}
S_{\alpha \alpha}\left(\tau_{k}\right)= & W\left(\tau_{k}\right)^{-1} \int \frac{1}{2}\left(3 \cos ^{2} \theta_{\alpha n}^{k}-1\right) \\
& \times \exp \left[-U_{\text {ext }}\left(\beta, \gamma, \tau_{k}\right) / k_{B} T\right] \sin \beta d \beta d \gamma
\end{aligned}
$$

with

$$
W\left(\tau_{k}\right)=\int \exp \left[-U_{\text {ext }}\left(\beta, \gamma, \tau_{k}\right) / k_{B} T\right] \sin \beta d \beta d \gamma,
$$

where $\theta_{\alpha n}^{k}$ is the angle between the director and principal axis $\alpha$ when the molecule is in the conformation specified by the values of the set $\tau_{k}$.

Making the division shown in Eq. (10) allows a conformational distribution, $P_{\text {iso }}\left(\tau_{k}\right)$, to be defined as

$$
P_{\text {iso }}\left(\tau_{k}\right)=Q^{-1} \exp \left[-U_{\text {int }}\left(\tau_{k}\right) / k_{B} T\right]
$$

with

$$
Q=\int \exp \left[-U_{\text {int }}\left(\tau_{k}\right) / k_{B} T\right] d \tau_{k} .
$$

This distribution is for the same temperature, and the same solvent as $P_{\mathrm{LC}}\left(\tau_{k}\right)$, but is for an isotropic phase. Note that $P_{\text {iso }}\left(\tau_{k}\right) \neq P_{\mathrm{LC}}\left(\tau_{k}\right)$, but that these distributions are related by

$$
P_{\text {iso }}\left(\tau_{k}\right)=P_{\mathrm{LC}}\left(\tau_{k}\right)\left\{Z /\left[W\left(\tau_{k}\right) Q\right]\right\} .
$$

\section{B. The additive potential (AP) model for $\boldsymbol{U}_{\text {ext }}\left(\boldsymbol{\beta}, \gamma, \tau_{k}\right)$}

There have been several suggestions as to how to model $U_{\text {ext }}\left(\beta, \gamma, \tau_{k}\right) .{ }^{14,15}$ For flexible molecules in particular there are advantages in using a general expansion in spherical harmonics, and for a uniaxial phase this is

$$
U_{\text {ext }}\left(\beta, \gamma, \tau_{k}\right)=-\sum_{\substack{L, m \\ \text { even }}}^{\infty} \varepsilon_{L, m}\left(\tau_{k}\right) C_{L, m}(\beta, \gamma) .
$$

The $C_{L, m}(\beta, \gamma)$ are modified spherical harmonics, and the $\varepsilon_{L, m}\left(\tau_{k}\right)$ are conformationally-dependent coefficients whose magnitudes depend upon the strength of the anisotropic part of the solute-solvent interaction. Computer simulations of solutes in liquid crystalline solvents suggest ${ }^{16}$ that the expansion in Eq. (18) can be restricted to $L=2$, and $m=0, \pm 2$ when the aim is to use it to obtain averages of second-rank interactions, such as dipolar or quadrupolar couplings. In the version of the AP method used here the $\varepsilon_{L, m}\left(\tau_{k}\right)$ will be approximated as sums of contributions $\varepsilon_{L, m}(j)$ from rigid molecular subunits,

$$
\varepsilon_{2, m}\left(\tau_{k}\right)=\sum_{p, j} \varepsilon_{2, p}(j) D_{p, m}^{2}\left(\Omega_{j}\left(\tau_{k}\right)\right)
$$




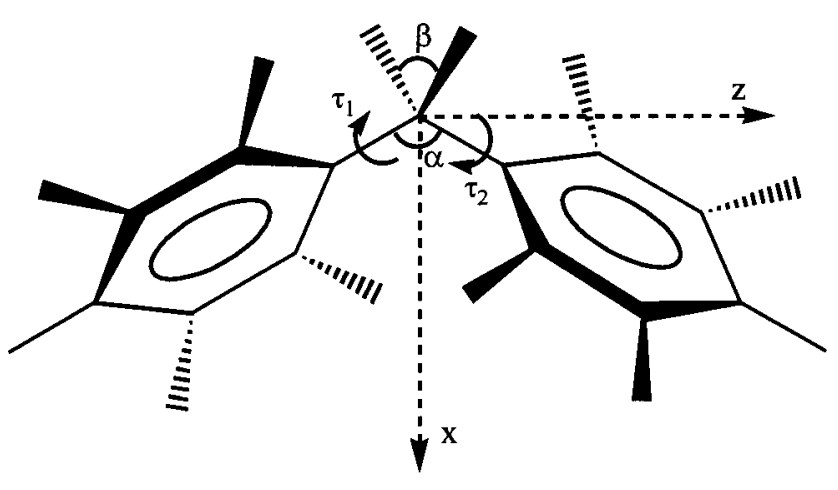

\begin{tabular}{cc}
\hline Conformation & Point Group \\
\hline$\tau_{1}=\tau_{2}=0^{\circ}, 90^{\circ}$ & $\mathrm{C}_{2 \mathrm{v}}$ \\
$\tau_{1}=0^{\circ}, \tau_{2}=90^{\circ}$ & $\mathrm{C}_{\mathrm{S}}$ \\
$\tau_{1}=-\tau_{2}$ & $\mathrm{C}_{\mathrm{S}}$ \\
$\tau_{1}=\tau_{2}$ & $\mathrm{C}_{2}$ \\
$\tau_{1} \neq \tau_{2}$ & $\mathrm{C}_{1}$ \\
\hline
\end{tabular}

FIG. 5. Conformations of diphenylmethane and their symmetry point groups.

where $D_{p, m}^{2}\left(\Omega_{j}\left(\tau_{k}\right)\right)$ is the Wigner function describing the orientation of fragment $j$ in molecular reference axes.

\section{Modelling the internal potential $U_{\text {int }}\left(\tau_{k}\right)$ as a truncated Fourier series}

There are also advantages in expanding $U_{\text {int }}\left(\tau_{k}\right)$ in a general way, and the most commonly adopted choice is as a truncated Fourier series, such as

$$
U_{\mathrm{int}}\left(\tau_{k}\right)=\sum_{l, n} V_{n} \cos \left(n \tau_{l}\right),
$$

with $n$ taking values to give the correct periodicity of $U_{\text {int }}\left(\tau_{k}\right)$, and the series being kept as short as will represent the correct overall shape of this bond rotational potential (at least near to the expected potential minima). However, for molecules like diphenylmethane this simple form for $U_{\text {int }}\left(\tau_{k}\right)$ is not able to describe correctly the energy surface. Thus, with $n=2$ and 4, which allows for energy minima with $0^{\circ}<\tau_{1}, \tau_{2}<90^{\circ}$, Eq. (20) predicts equal energies for structures with symmetry $C_{2}$ and $C_{s}$ (see Fig. 5). This energy degeneracy can be removed by adding additional terms. Thus, adding a term to account for short-range repulsion, which depends on $r_{i j}$, differentiates between the structures with $C_{2}$ and $C_{s}$ symmetries, but by only a small amount that could be insufficient for diphenylmethane. A more flexible approach is to add terms to Eq. (20) which depend on $\tau_{+}$ $=\tau_{1}+\tau_{2}$ and $\tau_{-}=\tau_{1}-\tau_{2}$,

$$
\begin{aligned}
U_{\mathrm{int}}\left(\tau_{1}, \tau_{2}\right)= & V_{2}\left(\cos 2 \tau_{1}+\cos 2 \tau_{2}\right) \\
& +V_{4}\left(\cos 4 \tau_{1}+\cos 4 \tau_{2}\right) \\
& +V_{+} \cos 2 \tau_{+}+V_{-} \cos 2 \tau_{-} .
\end{aligned}
$$

This gives an energy surface of the correct periodicity and symmetry, but it has the disadvantage shared by Fourier expansions for $U_{\mathrm{int}}\left(\tau_{k}\right)$ that it is not possible to vary the position of the energy minima, their relative energies, and the barriers between them in an independent way. This disadvantage can be overcome by adding more terms to the expansion, when fitting a functional form to the results from a calculation of an energy surface. Adding extra terms to Eq. (21) is usually not feasible when fitting observed data, such as averaged dipolar couplings, to a model for a probability distribution as in Eqs. (6), (8), and (10), because of the limited amount of experimental data available.

\section{The direct probability description of conformational distributions}

An alternative is to determine the probability $P_{\mathrm{LC}}\left(\tau_{k}\right)$ directly without going through the intermediate stage of first characterizing an energy surface. Such a probabilistic approach was proposed initially by Zannoni ${ }^{17}$ for obtaining $P_{\mathrm{LC}}\left(\beta, \gamma, \tau_{k}\right)$ from averaged NMR data. The original Zannoni method derived a form for $P_{\mathrm{LC}}\left(\beta, \gamma, \tau_{k}\right)$ by applying the maximum entropy (ME) principle. This gives

$$
P_{\mathrm{LC}}^{\mathrm{ME}}\left(\beta, \gamma, \tau_{k}\right)=Z_{\mathrm{ME}}^{-1} \exp \left[-U_{\mathrm{ME}}\left(\beta, \gamma, \tau_{k}\right) / k_{B} T\right]
$$

with

$$
Z_{\mathrm{ME}}=\int \exp \left[-U_{\mathrm{ME}}\left(\beta, \gamma, \tau_{k}\right) / k_{B} T\right] \sin \beta d \beta d \gamma d \tau_{k} .
$$

$U_{\mathrm{ME}}\left(\beta, \gamma, \tau_{k}\right)$ has the same form as $U_{\text {ext }}\left(\beta, \gamma, \tau_{k}\right)$ in Eq. (18) except that the coefficients depend directly on the experimental data and hence contain information on both the orientational order and the conformational distribution. This has the advantage of not going beyond the data available, and hence has been described as being the least biased approach. The method has the disadvantage however that $P_{\mathrm{LC}}\left(\beta, \gamma, \tau_{k}\right)$ in the isotropic phase becomes a constant independent of both orientational order, as it should, and conformation, which it should not. This disadvantage can be removed by introducing the constraint that $P_{\mathrm{LC}}\left(\beta, \gamma, \tau_{k}\right)$ become identical with $P_{\text {iso }}\left(\tau_{k}\right)$ in the isotropic phase. ${ }^{18,19}$

The method proposed here offers a different vision of the probabilistic approach. The distribution function $P_{\mathrm{LC}}\left(\tau_{k}\right)$ is modeled directly as a sum (for interdependent conformations) or product (for independent states) of Gaussian functions. Thus, for rotation about the $l$ th bond between $N_{l}$ conformations,

$$
P_{\mathrm{LC}}\left(\tau_{l}, N_{l}, k_{l_{j}}, \tau_{l_{j}}^{0}\right)=\sum_{j=1}^{N_{l}} A_{l_{j}} \exp \left[-\left\{k_{l_{j}}\left(\tau_{l}-\tau_{l_{j}}^{0}\right)\right\}^{2}\right]
$$

where the $\tau_{l_{j}}^{0}$ are the positions of maximum probability, $A_{l_{j}}$ are their relative amplitudes, and $k_{l_{j}}$ gives the width of the distribution. The physical interpretation is that the molecule exists in $j$ conformations, centered on $\tau_{l_{j}}^{0}$, and that there is a symmetric oscillation at each. The relative amounts of each conformer are obtained by integration. 


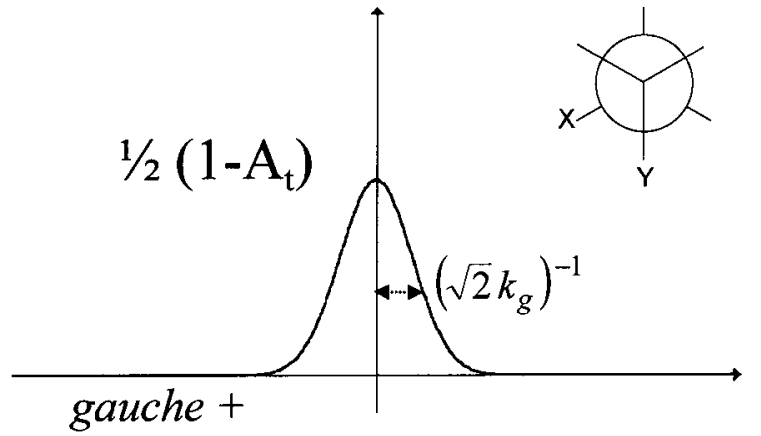

(a)

$$
\tau=\tau_{\mathrm{g}}
$$

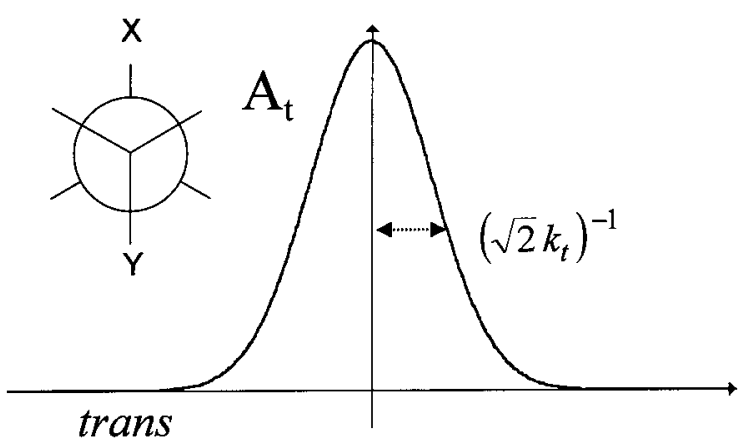

(b)

$$
\tau=0^{\circ}
$$

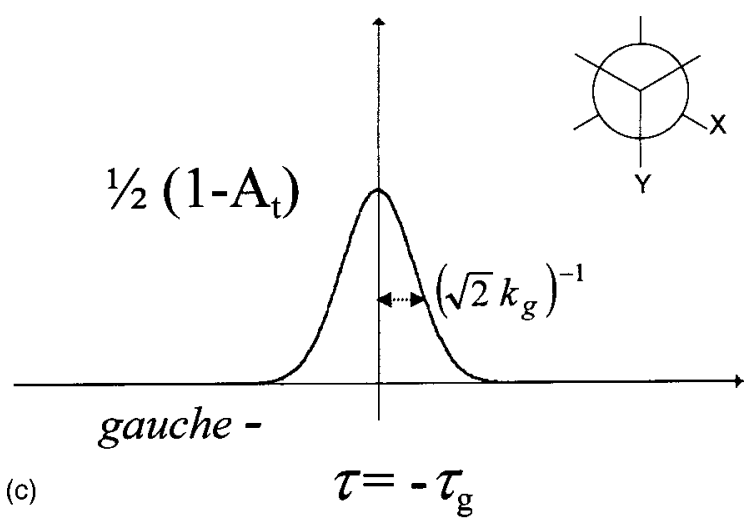

FIG. 6. The three Gaussian functions used to describe the three conformations generated by rotation about the $\mathrm{C}-\mathrm{C}$ bond in a compound of type $\mathrm{XCH}_{2} \mathrm{CH}_{2} \mathrm{Y}$.

A simple example is rotation about the $\mathrm{C}-\mathrm{C}$ bond in compounds of the type $\mathrm{XCH}_{2} \mathrm{CH}_{2} \mathrm{Y}$, as shown in Fig. 6. The variables used to describe this situation are $\tau_{g}, k_{g}, k_{t}$, and $A_{t}$; these can be varied independently, but with the constraint that $P_{\mathrm{LC}}\left(\tau, j, k_{j}, \tau_{j}\right)$ is normalized. We will return to a more detailed description of this case, with a comparison to the conventional cosine expansion of a potential energy function, in the future.

\section{APPLICATION OF THE PROBABILISTIC APPROACH TO DIPHENYLMETHANE}

The diphenylmethane molecules in the crystalline state are asymmetric in that the angles between the ring normals and the $\mathrm{HCH}$ plane are $\tau_{1}=63.9^{\circ}$ and $\tau_{2}=71.1^{\circ}$. The asymmetry must arise because of the asymmetric environment in the solid state, and is not expected either for an isolated molecule, or for an achiral liquid phase. Quantum chemistry calculations on an isolated diphenylmethane molecule $e^{6,7}$ suggest that only the structures with $C_{2}$ symmetry are appreciably populated. The probability function, $P_{\mathrm{LC}}\left(\tau_{1}, \tau_{2}\right)$ describing these conformations is conveniently expressed by introducing the angular variables $\tau_{+}=\tau_{1}+\tau_{2}$ and $\tau_{-}=\tau_{1}-\tau_{2}$,

$$
P_{\mathrm{LC}}\left(\tau_{+}, \tau_{-}\right)=P_{+}\left(\tau_{+}\right) P_{-}\left(\tau_{-}\right) .
$$

The correct periodicity of $P_{\mathrm{LC}}\left(\tau_{+}, \tau_{-}\right)$is obtained by introducing indices $s, n$, and $j$,

$$
\begin{aligned}
P_{\mathrm{LC}}\left(\tau_{+}, \tau_{-}\right)= & \sum_{s=0,1,2} \sum_{n=-1 / 2,1 / 2} \sum_{j=-1 / 2,1 / 2} \frac{1+s(2-s)}{2} \\
& \cdot \exp \left[-x_{+}^{2} / 2 h_{+}^{2}\right] \exp \left[-x_{-}^{2} / 2 h_{-}^{2}\right]
\end{aligned}
$$

with

$$
x_{+}=\tau_{+}+2 j\left(s \pi-\tau_{+}^{0}\right)
$$

and

$$
x_{-}=\tau_{-}+2 \operatorname{sn} \pi(2-s) .
$$

The maximum probability is constrained to have $\tau_{-}=0^{\circ}$ by the $C_{2}$ symmetry requirement, and to be at positions along $\tau_{+}$determined by $\tau_{+}^{0}$.

The value of $h_{+}$gives the full width at half maximum (FWHM) of the Gaussian function (related to the amplitude of conformational oscillation in the $\tau_{+}$direction) whilst $h_{-}$ is the same along $\tau_{-}$. The maps represented in Fig. 7 show how the shape of the distribution $P_{\mathrm{LC}}\left(\tau_{+}, \tau_{-}\right)$depends on the relative values of $h_{+}$and $h_{-}$.

The AP method was used to model $U_{\text {ext }}\left(\beta, \gamma, \tau_{1}, \tau_{2}\right)$ with identical contributions $\varepsilon_{z z}^{R}$ and $\varepsilon_{x x}^{R}-\varepsilon_{y y}^{R}$ from each phenyl ring. These were varied, together with $h_{+}, h_{-}, \tau_{+}^{0}$, and any appropriate geometrical parameters, to minimize the target function

$$
R=\left\{M^{-1} \sum_{i<j}\left[D_{i j}(\text { observed })-D_{i j}(\text { calculated })\right]^{2}\right\}^{1 / 2}
$$

with $M=$ number of independent couplings.

\section{RESULTS AND DISCUSSION}

The calculations proceeded first with the neglect of the effect on the $D_{i j}$ of small amplitude vibrational motion. The first step was to adjust the positions of protons in the aromatic rings to minimize $R$ for the intraring $D_{i j}$. The spectral analysis produces $D_{19,23}=D_{20,22}\left(=D_{15,17}\right)$, and so the distances $r_{19,23}, r_{20,22}$, and $r_{15,17}$ must be equal. Fixing the ring geometry to have:

all $r_{\mathrm{CC}}=1.40 \AA$, except $r_{12,13}\left(=r_{6,7}=r_{9,10}=r_{3,4}\right)$;

all CCC angles $120^{\circ}$ in the rings;

all $r_{\mathrm{CH}}=1.085 \AA$ except $r_{11,21}\left(=r_{5,16}\right)$;

the function $R$ was minimized by varying $\varepsilon_{z z}^{R}, \varepsilon_{x x}^{R}-\varepsilon_{y y}^{R}$ (or 

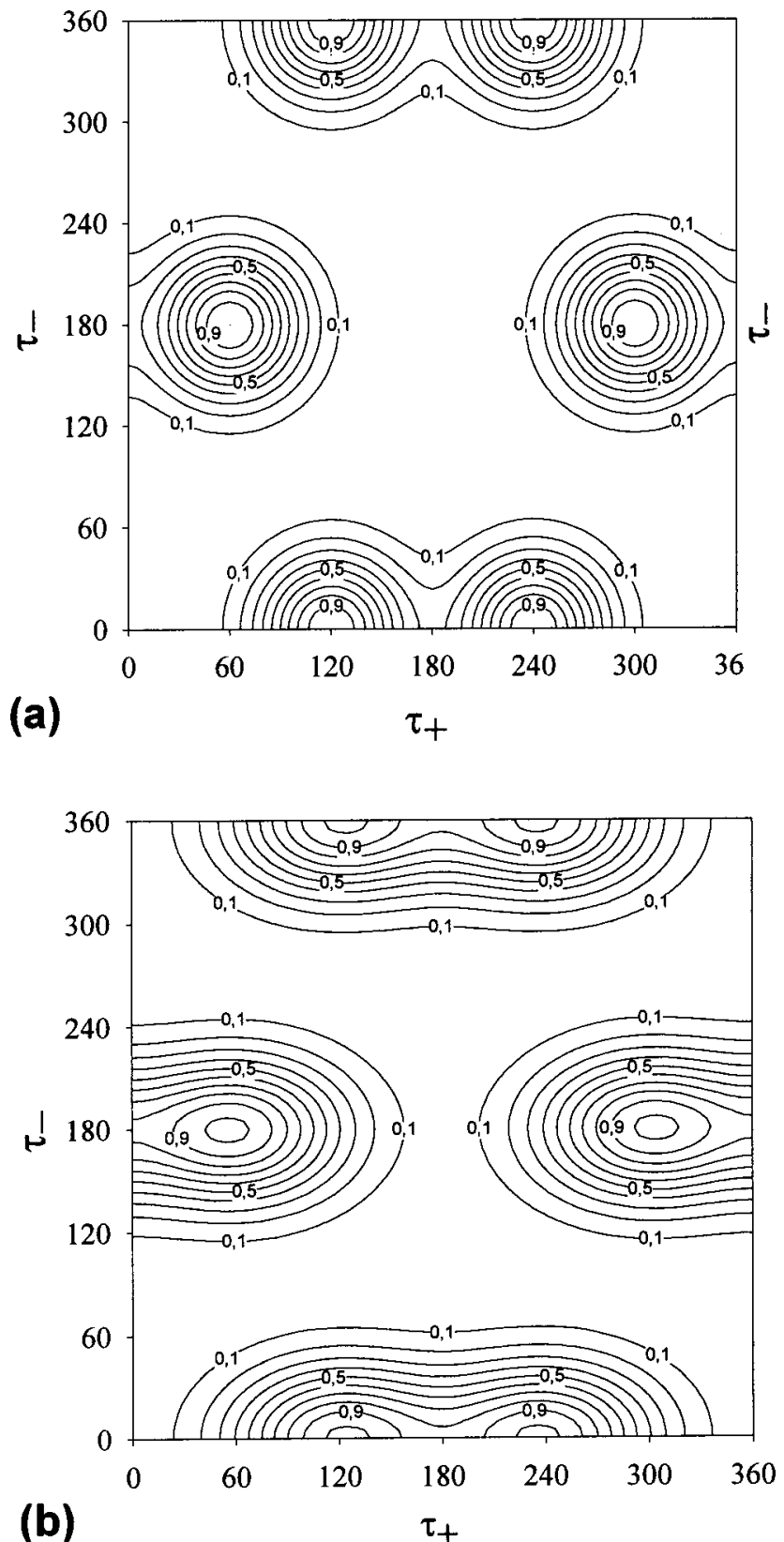

FIG. 7. The effect of $h_{+}$and $h_{-}$on the distribution $P_{\mathrm{LC}}\left(\tau_{+}, \tau_{-}\right)$; (a) has $h_{+}=h_{-}$, and in (b) $h_{+}=1.5 h_{-}$. The value of $\tau_{+}^{0}$ is the same in both cases.

TABLE III. Optimized and assumed geometrical parameters for diphenylmethane- $d_{3}$ (compound $\mathbf{3}$ ) and local order parameters, $S_{\alpha \beta}^{R}$, for the aromatic ring.

\begin{tabular}{|c|c|}
\hline Parameters & Optimized values \\
\hline$r_{9,10} / \AA$ & $1.398 \pm 0.002$ \\
\hline$r_{11,21} / \AA$ & $1.080 \pm 0.001$ \\
\hline$S_{c c}^{R}$ & $0.1140 \pm 0.0001$ \\
\hline \multirow[t]{2}{*}{$S_{a a}^{R}-S_{b b}^{R}$} & $0.1336 \pm 0.0001$ \\
\hline & Assumed values ${ }^{a}$ \\
\hline$\overline{\mathrm{CH}}$ (ring)/Å & 1.085 \\
\hline$\overline{\mathrm{CC}}$ (ring)/Å & 1.400 \\
\hline Angles (ring)/Å & 120.0 \\
\hline$r_{1,2} / \AA$ & 1.521 \\
\hline
\end{tabular}

${ }^{a}$ Taken from Ref. 22 and kept fixed.

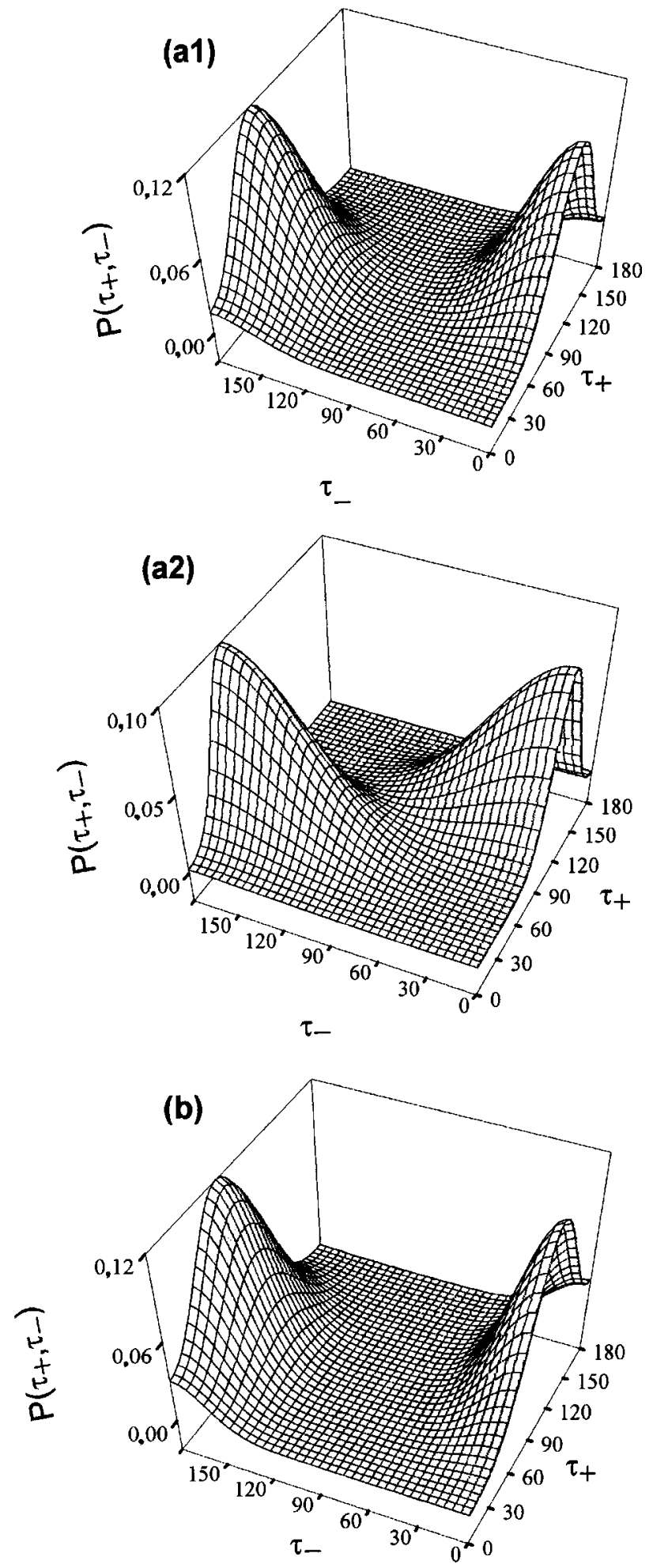

FIG. 8. The distribution $P_{\mathrm{LC}}\left(\tau_{+}, \tau_{-}\right)$determined by the probabilistic approach: (a1) and (a2) without, and (b) with vibrational corrections to the dipolar couplings (see text for explanation).

equivalently $S_{z z}^{R}$ and $S_{x x}^{R}-S_{y y}^{R}$ ), $r_{9,10}$ and $r_{11,21}$ to give the results shown in Table III. The ring geometries were then kept fixed in all subsequent calculations.

The potential energy surface for diphenylmethane has been calculated by a number of computational methods, ${ }^{6,7}$ and it was decided to investigate how well two such surfaces fit the observed dipolar couplings. In both cases the first 
TABLE IV. The values of the total rms error, $R$, and the geometrical parameters obtained by fitting the observed dipolar couplings with the potential energy surface calculated from MNDO and B3LYP/6-31G*.

\begin{tabular}{lcccccc}
\hline \hline & \multicolumn{3}{c}{ MNDO } & \multicolumn{4}{c}{ B 3LYP/6-31G* } \\
\hline$\alpha /^{\circ}$ & $114.7^{\mathrm{a}}$ & $126.3 \pm 0.5$ & $104.4 \pm 0.5$ & $114.7^{\mathrm{a}}$ & $118.6 \pm 0.5$ & $119.5 \pm 0.5$ \\
$\beta /^{\circ}$ & $105.2^{\mathrm{a}}$ & $105.2^{\mathrm{a}}$ & $124.7 \pm 0.5$ & $105.2^{\mathrm{a}}$ & $105.2^{\mathrm{a}}$ & $118.3 \pm 0.5$ \\
$R / \mathrm{Hz}$ & 84.7 & 29.7 & 17.3 & 91.9 & 5.1 & 4.7 \\
\hline \hline
\end{tabular}

${ }^{a}$ Taken from Ref. 22 and kept fixed.

calculations used the same geometry as the quantum chemistry calculations, and the only variables were $\varepsilon_{z z}^{R}$ and $\varepsilon_{x x}^{R}$ $-\varepsilon_{y y}^{R}$. The values of $R$ obtained, given in Table IV, are unacceptably large, and so the effect of retaining the calculated energy surface but varying the geometry was explored. The results are also shown in Table IV, and again are unacceptable. Note that when the $R$ value is not very high the value of the $\beta$ or $\alpha$ angles are unacceptable.

Fitting the $D_{i j}$ by the probabilistic approach, varying $\varepsilon_{z z}^{R}, \varepsilon_{x x}^{R}-\varepsilon_{y y}^{R}, \tau_{+}^{0}, h_{+}, h_{-}$, and the angle $\alpha$ gives a close fit to the data and the parameters shown in Table V, column A. In order to reduce the CPU requirements while keeping an high resolution $\left(1^{\circ}\right)$ in exploring the conformational surface, the calculations have been carried out by implementing a "smart," properly normalizing algorithm which catches only the essence of the distribution, without redundancies. It varies step by step $\tau_{1}$ from $0^{\circ}$ to $90^{\circ}$ and $\tau_{2}$ from $-\tau_{1}$ to $+\tau_{1}$. The obtained $D_{i j}$ are reported in Table II column A and the probability $P_{\mathrm{LC}}\left(\tau_{+}, \tau_{-}\right)$obtained is shown in Fig. 8(a1). It has a maximum at $\tau_{1}=\tau_{2}=56.5^{\circ} \pm 0.5^{\circ}$, compared with $57.0^{\circ}$ (Ref. 6) obtained by the B3LYP/6-31G* calculation, and a rather flat surface, as seen by the large values of $h_{+}$ $=29.5^{\circ}$ and $h_{-}=28.7^{\circ}$. The optimized value of $\alpha$ of $121.8^{\circ} \pm 0.5^{\circ}$ is significantly larger than that of $114.6^{\circ}$ determined by the B3LYP/6-31G* method. ${ }^{6}$

In Ref. 6, a strong correlation between $\alpha$ and $\tau_{1}$ and $\tau_{2}$ was found, and the calculations with this theoretical relationship were repeated to give the data of column B in Table V. Note that the value of $R$ is only slightly increased and the position of the maximum probability changes by only $2.8^{\circ}$ $\left(\tau_{1}=\tau_{2}=59.3^{\circ} \pm 0.5^{\circ}\right)$. The resulting distribution, shown in Fig. 8(a2), has quite different cross sections, the widths $h_{+}$ and $h_{-}$differing noticeably $\left(h_{+}=20.5^{\circ} ; h_{-}=47.4^{\circ}\right)$ from the previous ones.

\section{A. The effect of allowing for vibrational averaging of the dipolar couplings}

The inclusion of vibrational averaging into the derivation of $P_{\mathrm{LC}}\left(\tau_{+}, \tau_{-}\right)$is not straightforward. The main problem is the inclusion of small-amplitude vibrational motion in addition to the large amplitude bond rotational motion. This is a general problem, but it is compounded in the case of averaging of dipolar or quadrupolar couplings by having to include the variation of the orientational order as the molecules changes shape during these two kinds of motion. An approximate method is used for diphenylmethane, which is described more fully by Lesot $e t$ al. ${ }^{20}$ This method consists in calculating a wave function for small amplitude, harmonic vibrational motion for the molecule in the conformation with $\tau_{1}=\tau_{2}=56.5^{\circ}$ by the B3LYP/6-31G* method using the GAUSSIAN 98 package. ${ }^{21}$ This is then used to calculate vibration corrections to the $D_{i j}$, assuming that the order parameters are independent of the vibrational motion. The effect of the additional averaging over the large amplitude bond rotational motion is then done using the probabilistic method in conjunction with the AP method for calculating how the ordering changes with this motion.

The ring geometry was first optimized with the inclusion of vibrational averaging. This produced $r_{11,21}=1.074$ $\pm 0.002 \AA$, and $r_{9,10}=1.403 \pm 0.001 \AA$, which are changed by $<0.6 \%$. Then, using all the vibrational modes and varying $\varepsilon_{z z}^{R}, \varepsilon_{x x}^{R}-\varepsilon_{y y}^{R}, h_{+}, h_{-}, \tau_{+}^{0}$, and $\alpha$ produced the results shown in Table $\mathrm{V}$ column $\mathrm{C}$. The error, $R$, is considerably worse than without vibrational averaging. The value of $\tau_{+}^{0}$ is not changed significantly, corresponding to $\tau_{1}=\tau_{2}=58.8^{\circ}$ $\pm 0.5^{\circ}$.

The larger value of $R$ is probably because the effect of an oscillation involving $\tau_{1}$ and $\tau_{2}$ is counted twice: once as a

TABLE V. The interaction coefficients, $\varepsilon_{2, m}$, and the parameters obtained by fitting observed dipolar couplings with the probabilistic approach without vibrational corrections: optimizing the $\alpha$ angle (column A) and imposing to the $\alpha$ angle the behavior found from the B3LYP/6-31G* calculation (column B); and with vibrational contributions: using all the frequencies (column C) and without torsional frequencies (column D).

\begin{tabular}{lcccc}
\hline \hline & $\mathrm{A}$ & $\mathrm{B}$ & $\mathrm{C}$ & $\mathrm{D}$ \\
\hline$\varepsilon_{z z} / \mathrm{kJ} \mathrm{mol}^{-1}$ & $0.926 \pm 0.001$ & $0.881 \pm 0.001$ & $0.882 \pm 0.001$ & $0.915 \pm 0.001$ \\
$\varepsilon_{x x}-\varepsilon_{y y} / \mathrm{kJ} \mathrm{mol}^{-1}$ & $1.286 \pm 0.001$ & $1.292 \pm 0.001$ & $1.277 \pm 0.001$ & $1.274 \pm 0.001$ \\
$h_{+} /^{\circ}$ & $29.5 \pm 0.1$ & $20.5 \pm 0.7$ & $28.0 \pm 0.5$ & $34.7 \pm 0.1$ \\
$h_{-} /^{\circ}$ & $28.7 \pm 1.5$ & $47.4 \pm 0.4$ & $25 \pm 19$ & $21.3 \pm 3.7$ \\
$\tau_{+}^{0} /^{\circ}$ & $113.0 \pm 1.0$ & $118.6 \pm 1.0$ & $120.0 \pm 1.0$ & $110.2 \pm 1.0$ \\
$\alpha / l^{\circ}$ & $121.8 \pm 0.5$ & & $120.7 \pm 0.5$ & $121.3 \pm 0.5$ \\
$R / \mathrm{Hz}$ & 0.4 & 1.0 & 6.5 & 1.0 \\
\hline \hline
\end{tabular}


vibrational mode, and second by allowing the widths of the gaussian functions to vary. Note, too, the very large error on $h_{-}$in column $\mathrm{C}$ of Table $\mathrm{V}$, which is another indication that this model is flawed. Excluding the vibrational modes strongly dependent on $\tau_{1}$ and $\tau_{2}$ from the calculation of the vibrational average leads to $R=1.0 \mathrm{~Hz}$ and the results in column D of Table V. A value of $\tau_{1}=\tau_{2}=55.1 \pm 0.5^{\circ}$ is now obtained. There is a large change in the values of $h_{+}$and $h_{-}$, which is reflected in a large change in the shape of the distribution $P_{\mathrm{LC}}\left(\tau_{1}, \tau_{2}\right)$ as shown in Fig. 8(b).

\section{B. Using a jump model for $P_{\mathrm{LC}}\left(\tau_{1}, \tau_{2}\right)$}

It is often assumed that only conformations corresponding to the positions of energy minima need to be considered when averaging dipolar or quadrupolar couplings. In the probabilistic model this corresponds to collapsing the Gaussians into delta functions at the positions $\tau_{+}^{0}$. This approximation was explored, including averaging over all the vibrational modes, the torsional ones too in order to simulate the torsion as a libration about the minimum. In this way a minimum in $R$ of $19 \mathrm{~Hz}$ was found when $\tau_{1}=\tau_{2}=70^{\circ}$. Allowing for a change of the $\mathrm{HCH}$ angle the value of $R$ is reduced to $1 \mathrm{~Hz}$, but we obtain for that angle an unacceptable value of $93^{\circ}$.

\section{Calculation of $P_{\text {iso }}\left(\tau_{1}, \tau_{2}\right)$}

Having obtained $P_{\mathrm{LC}}\left(\tau_{1}, \tau_{2}\right)$ directly from the experimental values of the $D_{i j}$ it is straightforward to calculate the distribution $P_{\text {iso }}\left(\tau_{1}, \tau_{2}\right)$ from Eq. (17). These distributions are expected to differ when the $\varepsilon_{2, n}\left(\tau_{k}\right)$ have a strong dependence on conformation. This corresponds to the conformations having very different shapes. Thus, differences of up to $30 \%$ were found for the liquid crystal $5 \mathrm{CB} .{ }^{14}$ For diphenylmethane, however, the differences are found to be small $(<3 \%)$.

\section{CONCLUSION}

The application of the probabilistic approach, coupled with the AP model for the conformational dependence of the interaction with the liquid crystal molecules, gives a distribution $P_{\mathrm{LC}}\left(\tau_{1}, \tau_{2}\right)$ which is similar to that found by quantum chemical calculations. The positions of the maxima in the distribution are in particularly close agreement. This cannot be taken to be a proof of the validity of the model used, but it suggests that this simple method of representing $P_{\mathrm{LC}}\left(\tau_{1}, \tau_{2}\right)$ in terms of Gaussian functions is a good approximation.

\section{ACKNOWLEDGMENTS}

This work has been supported by MIUR PRIN ex $40 \%$. The authors gratefully acknowledge Dr. Rosa Saladino for her help in the spectral analysis and Dr. J. Grunenberg for his "remote" support and kindness during the progress of the work. J.W.E. thanks the Leverhulme Trust for the award of an Emeritus Fellowship.

${ }^{1}$ J. Kaski, J. Vaara, and J. Jokisaari, J. Am. Chem. Soc. 118, 8879 (1996).

${ }^{2}$ E. de Alba and N. Tjandra, Prog. Nucl. Magn. Reson. Spectrosc. 40, 175 (2002).

${ }^{3}$ I. Bertini, C. Luchinat, and G. Parigi, Prog. Nucl. Magn. Reson. Spectrosc. 40, 249 (2002).

${ }^{4}$ R. Mahjan, A. Vora, and A. Pathak, in Proceedings of the ILCC 2002, Edinburgh, 30 June-5 July 2002, p. 187.

${ }^{5}$ J. C. Barnes, J. D. Paton, J. R. Damewood, Jr., and K. Mislow, J. Org. Chem. 46, 4975 (1981).

${ }^{6}$ M. Feigel, J. Mol. Struct. 366, 83 (1996).

${ }^{7}$ T. Strassner, Can. J. Chem. 75, 1011 (1997).

${ }^{8}$ J. W. Emsley, G. R. Luckhurst, and C. P. Stockley, Mol. Phys. 44, 565 (1981).

${ }^{9}$ J. W. Emsley, in Solid-State NMR Spectroscopy Principles and Applications, edited by M. J. Duer (Blackwell Science, New York, 2002), p. 531.

${ }^{10}$ M. Longeri, G. Chidichimo, and P. Bucci, Org. Magn. Reson. 22, 408 (1984).

${ }^{11}$ G. Celebre, G. De Luca, M. Longeri, and E. Sicilia, J. Chem. Inf. Comput. Sci. 34, 539 (1994).

${ }^{12}$ J. W. Emsley and G. R. Luckhurst, Mol. Phys. 41, 19 (1980).

${ }^{13}$ P. Diehl, in NMR of Liquid Crystals, edited by J. W. Emsley (Reidel, Dordrecht, 1985), Chap. 7.

${ }^{14}$ J. W. Emsley, G. R. Luckhurst, and C. P. Stockley, Proc. R. Soc. London, Ser. A 381, 117 (1982).

${ }^{15} \mathrm{~J}$. W. Emsley, in Encyclopedia of NMR, edited by D. M. Grant and R. K. Harris (Wiley, Chichester, 1995), p. 2781

${ }^{16}$ G. La Penna, E. K. Foord, J. W. Emsley, and D. J. Tildesley, J. Chem. Phys. 104, 233 (1996).

${ }^{17}$ C. Zannoni, in NMR of Liquid Crystals edited by J. W. Emsley (Reidel, Dordrecht, 1985), Chap. 2.

${ }^{18}$ D. Catalano, J. W. Emsley, G. La Penna, and C. A. Veracini, J. Chem. Phys. 105, 10595 (1996).

${ }^{19}$ R. Berardi, F. Spinozzi, and C. Zannoni, Chem. Phys. Lett. 260, 633 (1996)

${ }^{20}$ P. Lesot, D. Merlet, J. Courtieu, J. W. Emsley, T. T. Rantala, and J. Jokisaari, J. Phys. Chem. 101, 5719 (1997).

${ }^{21}$ M. J. Frisch, G. W. Trucks, H. B. Schlegel et al., GAUSSIAN 98, Revision A.9, Gaussian, Inc., Pittsburgh, PA, 1998.

${ }^{22}$ S. A. Katsyuba, J. Grunenberg, and R. Schmutzler, J. Mol. Struct. 559, 315 (2001). 